\title{
Linear Bénard-Marangoni instability in rigid circular containers
}

\author{
P. C. Dauby, ${ }^{*}$ G. Lebon, ${ }^{\dagger}$ and E. Bouhy \\ Université de Liège, Institut de Physique B5, Sart Tilman, B 4000 Liège 1, Belgium \\ (Received 15 October 1996; revised manuscript received 28 February 1997)
}

\begin{abstract}
A linear study of the Bénard-Marangoni instability in small rigid circular containers is presented. The fluid is assumed to be Newtonian with a temperature-dependent surface tension at the upper free surface. The layer is horizontal and heated from below. The principle of exchange of stability is numerically shown to be valid. The critical conditions for the onset of motion are determined as well as the convective patterns at the threshold. Generally, the cells take the form of either concentric or "transverse" rolls. The coupled influence of gravity and capillarity is analyzed and the influence of the gas lying above the fluid on the Biot number is also taken into account. Comparisons with other works are discussed. [S1063-651X(97)13607-8]
\end{abstract}

PACS number(s): 44.25.+f, 47.20.-k, 47.27.Te

\section{INTRODUCTION}

The problem of thermoconvective instabilities in fluid layers heated from below has become a classical subject in fluid mechanics. Many authors have analyzed this problem and the bibliography is very large. An interesting review can be found, for instance, in the book of Koschmieder [1].

It is well known that two different effects are responsible for the onset of motion when the temperature difference becomes larger than a certain threshold value. The first one is referred to as the Rayleigh-Bénard effect and originates in the gravity force. After the experiments of Bénard in 1900 [2], this effect was studied from a theoretical point of view by Rayleigh in 1916 [3]. The second possible cause of motion is the so-called Marangoni effect, which generates motion due to the capillary forces appearing at an upper free surface whose tension is a function of temperature. This effect was described theoretically by Pearson in 1958 [4]. In Earth grounded experiments, both effects combine and give rise to the "Bénard-Marangoni" instability studied in 1964 by Nield [5].

Until rather recently, most of the theoretical studies were concerned with layers of infinite horizontal extent and these approaches did not model lateral side-wall effects; the corresponding experiments were carried out in containers of very large horizontal extent for which the influence of lateral walls may actually be disregarded.

Experimental works on confined thermocapillary convection are today still a bit scarce. A very important paper in this area is the work of Koschmieder and Prahl [6] who reported careful experiments on Bénard-Marangoni instability in small square and circular containers. Other interesting experimental works are those of Ezersky et al. [7], Ondarçuhu et al. [8], and Ondarçuhu et al. [9]. A recent work by Johnson and Narayanan [10] presents experimental results for circular containers with aspect ratios equal to 1.5 and 2.5 and shows the possibility of oscillatory motion. The particu-

\footnotetext{
*Electronic address: PC.Dauby@ulg.ac.be

${ }^{\dagger}$ Also at Louvain University, Department of Mechanics, B 1348 Louvain-La-Neuve, Belgium.
}

lar case of slippery side walls was also studied experimentally in [11].

Despite this relative lack of experimental results, more and more theoretical studies are devoted nowadays to the thermoconvective instabilities in fluid layers of finite horizontal extent. A precursory theoretical work in this area is the paper of Davis in 1967 [12], who studied pure gravitydriven instability in rectangular containers with rigid horizontal and lateral walls. The problem of thermocapillary convection in finite boxes was first considered by Rosenblat, Davis, and Homsy [13] and Rosenblat, Homsy, and Davis [14]. Their studies are both linear and nonlinear but they use the restrictive assumption of vorticity-free lateral walls. This assumption has also been used by Dauby et al. [15] who analyzed hexagonal convective cells and also by Echebarría et al. [16] and Johnson and Narayanan [17]. Linear and nonlinear approaches to two-dimensional (2D) Marangoni convection in rigid rectangular containers have been examined by several authors using different numerical methods [1820].

The three-dimensional problem has been considered only recently. Three papers by Dijkstra [21-23] have been devoted to a rather complete study of Marangoni instability in square containers. The convective thresholds are determined and several bifurcation diagrams are given. Good agreement with experiments is obtained in small boxes. The appearance of hexagonal convective cells is also examined in larger containers. Another analysis of this problem was proposed in 1996 for rectangular cavities by Dauby and Lebon [24] who use amplitude equations to reduce the dynamics of the system to the dynamics of the most unstable modes of convection. Bifurcation diagrams are presented and the results are in very good agreement with all the experiments of Koschmieder and Prahl in square boxes with aspect ratio smaller than about 8 .

The case of circular containers has also been examined in several papers. In 1981, Vrentas et al. [25] studied pure gravity and pure capillarity-driven convection in circular vessels but their work is restricted to a 2D (axisymmetric) linear approach. In 1991, Chen et al. [26] considered the 3D linear Marangoni problem with an adiabatic upper surface but their results for axisymmetric perturbations seem a bit doubtful since they do not recover Nield's results in large 
aspect ratio boxes. A nonlinear code was also used by Wagner et al. [27] to determine the convective thresholds by interpolating the bifurcation points. The agreement between their results and those of the two above-mentioned papers is good since the deviation with the non-axisymmetric results of Chen et al. is about $6 \%$ while the difference with Vrentas et al. is less than $2 \%$.

Very recently, Zaman and Narayanan [28] proposed a linear study of gravity- and capillarity-driven convection in circular boxes. They determine the critical heating for the appearance of motion and give pictures of the convective patterns at the threshold. The agreement between this paper and the work of Vrentas et al. is quite good in the case of pure Rayleigh convection since the deviation between both results is about $2 \%$ for an aspect ratio equal to 1 and becomes still smaller in larger containers. For the Marangoni problem, the agreement is also quite good in large boxes (difference less than 2\%) but becomes less satisfactory in small aspect ratio vessels. Note also that these authors present a detailed comparison of their results for conducting lateral side walls with the experimental data of Koschmieder and Prahl [6].

In the present work, a similar linear analysis of the coupled gravity- and capillarity-driven instability is developed. However, our numerical method is different since we used a spectral tau method with Chebishev decompositions in both the $z$ and $r$ directions. Moreover our code works differently since it allows one to determine altogether the Rayleigh and Marangoni numbers defining criticality while Ra was fixed in Zaman and Narayanan's approach. We have also tried to model carefully the experimental situation used by Koschmieder and Prahl. In particular, we show that the Biot number to be considered in the calculations depends on the aspect ratio of the container.

Apart from these rather technical reasons, our paper is motivated by the following points. First, it is clear that the results of a linear analysis of thermoconvection in confined geometries must tend to Nield's results [5] corresponding to horizontally infinite domains when the aspect ratio gets larger and larger. In Zaman and Narayanan's paper, the critical numbers for an aspect ratio equal to 8 are within Nield's values by $1.2 \%$ but are smaller than these values. This fact is a bit astonishing since side walls should always play a stabilizing role. Another important point is the comparison between insulating and conducting side walls. For reasons discussed below, the critical heating for insulating side walls should always lie below that for conducting boundaries. The results of Zaman and Narayanan comply with this requirement in small boxes but not always in larger ones, even if the error is less than $1 \%$. For these two reasons, we have thought it interesting to reproduce the calculations. Moreover, an original by-product of our study is a numerical proof of the validity of the principle of exchange of stability for the coupled gravity- and capillarity-driven instability problem. Eventually, the comparison with the experiments of Koschmieder and Prahl is also very briefly discussed in the case of insulating side walls.

The content of the paper is as follows. In Sec. II, the basic equations are recalled very briefly since these are the same as in the work of Zaman and Narayanan. Section III and the Appendix consist of a description of the numerical method.
The results are then presented in Sec. IV. Eventually, our approach is compared with other works and with experiments in Sec. V and a short summary is presented in the last section.

\section{BASIC LINEAR EQUATIONS}

Let us consider a fluid layer of thickness $d$ filling a circular container with radius $a d$ ( $a$ is the aspect ratio). The surface tension at the upper free surface is temperature dependent and the fluid is heated from below. It is well known that motion sets in after the vertical temperature gradient has reached a critical value.

In the reference state, there is no motion and heat propagates by conduction only. In the linear Boussinesq equations, the azimuthal variable $\phi$ can be separated from $r$ and $z$. We will then seek a solution of the form

$$
\begin{gathered}
u(t, r, \phi, z)=u_{m}(t, r, z) \cos m \phi, \\
v(t, r, \phi, z)=\nu_{m}(t, r, z) \sin m \phi, \\
w(t, r, \phi, z)=w_{m}(t, r, z) \cos m \phi, \\
\theta(t, r, \phi, z)=\theta_{m}(t, r, z) \cos m \phi, \\
p(t, r, \phi, z)=p_{m}(t, r, z) \cos m \phi,
\end{gathered}
$$

where $\theta$ and $\mathbf{v}=u \mathbf{e}_{r}+v \mathbf{e}_{\theta}+w \mathbf{e}_{z}$ are the infinitesimal temperature and velocity perturbations with respect to the conductive solution, $p$ is the pressure perturbation, and $(r, \phi, z)$ are the polar coordinates. The $z$ axis is vertical and oriented from the bottom to the top of the box.

The general equations for the fields with index $m$ in Eq. (2.1) are easily shown to be

$$
\begin{gathered}
\frac{1}{a r} \frac{\partial(r u)}{\partial r}+\frac{m}{a r} v+\frac{\partial w}{\partial z}=0 \\
\operatorname{Pr}^{-1} \frac{\partial u}{\partial t}=-\frac{1}{a} \frac{\partial p}{\partial r}+\nabla_{m^{2}+1}^{2} u-\frac{2 m}{a^{2} r^{2}} v \\
\operatorname{Pr}^{-1} \frac{\partial v}{\partial t}=\frac{m}{a r} p+\nabla_{m^{2}+1}^{2} v-\frac{2 m}{a^{2} r^{2}} u \\
\operatorname{Pr}^{-1} \frac{\partial w}{\partial t}=-\frac{\partial p}{\partial z}+\nabla_{m^{2}+1}^{2} w+\operatorname{Ra} \theta \\
\frac{\partial \theta}{\partial t}=w+\nabla_{m^{2}}^{2} \theta
\end{gathered}
$$

where the index $m$ has been canceled for simplicity. These equations are written in dimensionless form with vertical distance, horizontal distance, time, and temperature scaled by $d, a d, d^{2} / \kappa$, and $\Delta T$, respectively; $\Delta T$ is the (conductive) temperature drop between the bottom and the top of the layer, and $p$ is the dimensionless pressure. The Rayleigh number is defined by $\mathrm{Ra}=\alpha_{T} g \Delta T d^{3} / \kappa \nu$, where $\alpha_{T}$ is the coefficient of volume expansion and $g$ the intensity of gravity; $\nu$ is the kinematic viscosity of the fluid and $\kappa$ its heat 
diffusivity. The Prandtl number is given by $\operatorname{Pr}=\nu / \kappa . \nabla_{n}^{2}$ is defined by $\nabla_{n}^{2}=a^{-2} r^{-1} \partial / \partial r(r \partial / \partial r)-n a^{-2} r^{-2}+\partial^{2} / \partial z^{2}$.

The boundary conditions are the following. The bottom of the box is rigid and assumed to be perfectly heat conducting so that

$$
\mathbf{v}=\theta=0 \quad \text { at } \quad z=0
$$

The upper surface of the fluid is assumed to be free, plane, and nondeformable. The surface tension $\xi$ is supposed to be a linear function of the temperature; the constant $\gamma=$ $-\partial \xi / \partial T$ will be considered positive, as is usual for most fluids. We also assume that, at the top surface, heat is transferred from the liquid to the ambient gas according to Newton's law of cooling, which results in a Biot condition for the temperature perturbations. The mathematical expressions of the boundary conditions at the upper surface are then $[4,5,12,13,28]$

$$
\begin{gathered}
w=0 \quad \text { at } \quad z=1, \\
\frac{\partial \theta}{\partial z}+\operatorname{Bi} \theta=0 \quad \text { at } \quad z=1, \\
\frac{\partial u}{\partial z}+\operatorname{Ma} \frac{1}{a} \frac{\partial \theta}{\partial r}=0 \quad \text { at } \quad z=1, \\
\frac{\partial v}{\partial z}-\operatorname{Ma} \frac{m}{a r} \theta=0 \quad \text { at } \quad z=1,
\end{gathered}
$$

where $\mathrm{Bi}$ is the Biot number and $\mathrm{Ma}=\gamma \Delta T d / \rho \kappa \nu$ is the Marangoni number with $\rho$ the constant mass density.

The lateral side wall is rigid and we will consider both conducting (CSW) and adiabatically insulated (ISW) side walls. The corresponding boundary conditions for velocity and temperature are

$$
\mathbf{v}=\mathbf{0}, \quad \partial \theta / \partial r=0 \quad(\mathrm{ISW}), \quad \theta=0 \quad(\mathrm{CSW}) \quad \text { at } \quad r=1 .
$$

Note eventually that the use of cylindrical coordinates, which are singular at $r=0$, imposes regularity conditions on the velocity and temperature fields [29]. These conditions express that the unknown fields are single valued at $r=0$. For the temperature and velocity perturbations, these conditions are written as

$$
\begin{gathered}
\theta=0 \quad \text { at } \quad r=0 \text { for } m \neq 0, \\
u=\nu=0 \quad \text { at } r=0 \text { for } m=0, \\
u=-\nu ; w=0 \text { at } r=0 \text { for } m=1, \\
u=\nu=w=0 \quad \text { at } \quad r=0 \text { for } m \neq 0,1 .
\end{gathered}
$$

\section{SOLUTION AND NUMERICAL METHOD}

In order to determine the critical temperature difference, an exponential time dependence of the form $\exp (\sigma t)$ for all the variables is introduced in the equations. The principle of exchange of stability is taken for granted here but we show later on that this hypothesis is actually valid. So the convec-
TABLE I. Convergence of the critical Marangoni number $\mathrm{Ma}_{c}$ with $N_{r}$ and $N_{z}$ for insulating side walls. The aspect ratio is equal to $5 ; \mathrm{Bi}=2$ and $\mathrm{Ra}=0$. The values of the azimuthal wave numbers are given in the first column. The next three columns give the values of $\mathrm{Ma}_{c}$ for different $N_{r} \times N_{z}$. The last column gives the difference in $\%$ between columns " $13 \times 9$ " and " $9 \times 5$."

\begin{tabular}{ccccc}
\hline \hline$N_{r} \times N_{z}$ & $9 \times 5$ & $11 \times 7$ & $13 \times 9$ & $\Delta_{13 \times 9-9 \times 5}(\%)$ \\
\hline$m=0$ & 154.1463 & 154.0648 & 154.0630 & 0.05 \\
$m=1$ & 152.9577 & 152.9518 & 152.9515 & 0.004 \\
$m=2$ & 154.3350 & 154.2497 & 154.2105 & 0.08 \\
$m=3$ & 153.2802 & 153.2720 & 153.2684 & 0.008 \\
$m=4$ & 153.9933 & 153.9915 & 153.9908 & 0.002 \\
\hline \hline
\end{tabular}

tion threshold is determined by evaluating the critical Marangoni and Rayleigh numbers corresponding to a zero growth rate $\sigma$. It is also worth stressing that the vertical and horizontal coordinates cannot be separated as in vorticityfree boxes [13]. For this reason, a numerical solution of the equations is required.

The numerical method used here is a spectral tau method [29], which is similar to the method used by Dauby and Lebon for the study of rectangular rigid containers [24]. The unknown fields are expanded in series of trial functions that form a complete set and satisfy some of the boundary conditions, the so-called "essential" boundary conditions. Truncated series are introduced in the field equations as well as in the "natural" boundary conditions, which are not a priori satisfied by the trial functions. Then these equations and natural boundary conditions are projected on the same trial functions; i.e., the equations and natural boundary conditions are multiplied by the trial functions before being integrated over the $(r, z)$-fluid volume.

In the present study, the velocity and temperature perturbations are written in the form

$$
\begin{aligned}
\left(\begin{array}{c}
\mathbf{v} \\
\theta
\end{array}\right)= & \sum_{i=1}^{N_{r}} \sum_{k=1}^{N_{z}} A_{i k}\left(\begin{array}{c}
\mathbf{v}_{i k}^{r} \\
0
\end{array}\right)+\sum_{i=1}^{N_{r}} \sum_{k=1}^{N_{z}} B_{i k}\left(\begin{array}{c}
\mathbf{v}_{i k}^{\theta} \\
0
\end{array}\right)+\sum_{k=1}^{N_{z}} X_{k}\left(\begin{array}{c}
\mathbf{v}_{k}^{0} \\
0
\end{array}\right) \\
& +\sum_{i=1}^{N_{r}} \sum_{k=1}^{N_{z}} C_{i k}\left(\begin{array}{c}
0 \\
\theta_{i k}
\end{array}\right)
\end{aligned}
$$

where $N_{r}$ and $N_{z}$ are integers; $\mathbf{v}_{i k}^{r}=\left(u_{i k}^{r}, 0, w_{i k}^{r}\right), \quad \mathbf{v}_{i k}^{\theta}$ $=\left(0, \nu_{i k}^{\theta}, w_{i k}^{\theta}\right), \mathbf{v}_{i k}^{0}=\left(u_{i k}^{0}, \nu_{i k}^{0}, w_{i k}^{0}\right)$, and $\theta_{i k}$ are trial functions, which are specified in the Appendix; $A_{i k}, B_{i k}, X_{k}$, and $C_{i k}$ are unknown constants. The pressure $p$ is not given an explicit decomposition because the pressure gradient disappears in the final equations as a result of integration by parts and the boundary conditions.

Before examining in detail the results of our study, it is important to consider the problem of the convergence of the calculations when $N_{r}$ and $N_{z}$ are increased. Table I presents the values of the critical Marangoni numbers calculated in the case of insulating side walls for different values of these two parameters. The aspect ratio is equal to 5 and $\mathrm{Bi}=2$. The Rayleigh number is zero. These values are selected to examine the convergence for the following reasons. The slowest convergence is obtained in large aspect ratio boxes since the convective pattern is then more complex. Similarly, large 
TABLE II. Critical Marangoni numbers $\mathrm{Ma}_{c}$ for insulating (ISW) and conducting (CSW) side walls and for different azimuthal wave number $m$. The Rayleigh and Biot numbers are 100 and 0.2 , respectively. The corresponding Nield's value for an infinite layer is equal to 75.544. For $a=8$, we used $N_{r} \times N_{z}=13 \times 7$.

\begin{tabular}{ccccccccc}
\hline \hline & \multicolumn{2}{c}{$a=1$} & \multicolumn{2}{c}{$a=2$} & \multicolumn{2}{c}{$a=4$} & \multicolumn{2}{c}{$a=8$} \\
\cline { 2 - 9 }$m$ & ISW & CSW & ISW & CSW & ISW & CSW & ISW & CSW \\
\hline 0 & 163.57 & 179.73 & 80.876 & 91.174 & 78.777 & 79.545 & 76.196 & 76.467 \\
1 & 109.08 & 198.06 & 91.249 & 94.459 & 77.870 & 79.326 & 76.487 & 76.504 \\
2 & 160.15 & 274.09 & 99.113 & 105.53 & 79.783 & 79.968 & 76.233 & 76.518 \\
3 & 257.86 & 390.67 & 101.20 & 123.50 & 78.141 & 79.902 & 76.531 & 76.531 \\
\hline \hline
\end{tabular}

Biot numbers make smaller cells (see below) and thus more complicated structures. The Rayleigh number does not have a great influence on the pattern (see below) and is then arbitrarily fixed to zero.

Table I shows that $N_{r} \times N_{z}=9 \times 5$ gives very good results for aspect ratios less than about 5 and Biot numbers less than about 2. So, except where otherwise stated, all results given below correspond to the values $N_{r} \times N_{z}=9 \times 5$.

\section{NUMERICAL RESULTS}

For possible checking, we present a sample of numerical results in Table II. Some interesting comments may be made about these results. First of all, the critical Marangoni numbers for finite boxes are always larger than the values corresponding to an infinite domain, which means that rigid side walls are stabilizing as expected. Moreover, $\mathrm{Ma}_{c}$ tends to Nield's values for large aspect ratios, whatever the value of the azimuthal wave number $m$ and for both conducting and insulating side walls. We also notice that the critical Marangoni numbers for insulating side walls are always smaller than for conducting ones. This is due to the fact that a temperature perturbation arriving at an insulating boundary is reflected towards the bulk of the fluid while it is dissipated in the walls when these are conducting. For this reason, conducting side walls give rise to more stable systems. This argument is similar to Nield's [5] who interpreted the increase of $\mathrm{Ma}_{c}$ with $\mathrm{Bi}$ in infinite layers.

It is also interesting to plot $\mathrm{Ma}_{c}$ as a function of the aspect ratio of the container. Results for insulating side walls are presented in Fig. 1 when $\mathrm{Ra}=\mathrm{Bi}=0$. The aspect ratio varies from 0.5 to 5 . The curves corresponding to values of $m$ between 0 and 3 are presented; the critical Marangoni number is the absolute minimum of these curves. Azimuthal wave numbers larger than 3 are not considered in the figures since these never correspond to the absolute minimum. We observe that the critical Marangoni number is a globally decreasing function of the aspect ratio of the box. Similar results were obtained by Dauby and Lebon in the case of square and rectangular containers [24]. The decrease of $\mathrm{Ma}_{c}$ consists of different portions of concave curves, each of which corresponds to a typical convective pattern. The different convective patterns are discussed in more detail below.

Despite its theoretical importance, it is worth noting that the "usual case" $\mathrm{Ra}=\mathrm{Bi}=0$ can never correspond to real experimental conditions. Actually, under terrestrial conditions, gravity is never completely negligible and $\mathrm{Ra}$ is not zero. Moreover, the zero Biot number condition at the top surface is also approximate. Note also that the adiabatic lateral wall assumption must also be considered with caution since the thermal condition on lateral walls may be of some importance in small containers.

For these reasons, we have tried to provide a better account of the real experimental conditions by modeling with some care the conditions under which the observations of Koschmieder and Prahl [6] were realized. Let us consider in particular the thermal condition at the upper free surface of the fluid layer. In the experiments, a $0.5-\mathrm{mm}$ air layer lies above the fluid and the temperature is kept fixed at the top of this air layer. In these circumstances, one can give the following approximate value for the Biot number at the upper free surface of the liquid [30]:

$$
\mathrm{Bi}=\frac{\lambda_{\text {air }}}{\lambda_{\mathrm{fl}}} \frac{k}{\tanh \left(k d_{\text {air }}\right)}
$$

In this formula, $\lambda_{\text {air }}$ and $\lambda_{\mathrm{ff}}$ represent the conductivities of the air and of the fluid; $d_{\text {air }}$ is the dimensionless thickness of the air layer, that is, $0.5 \mathrm{~mm}$ divided by the thickness of the

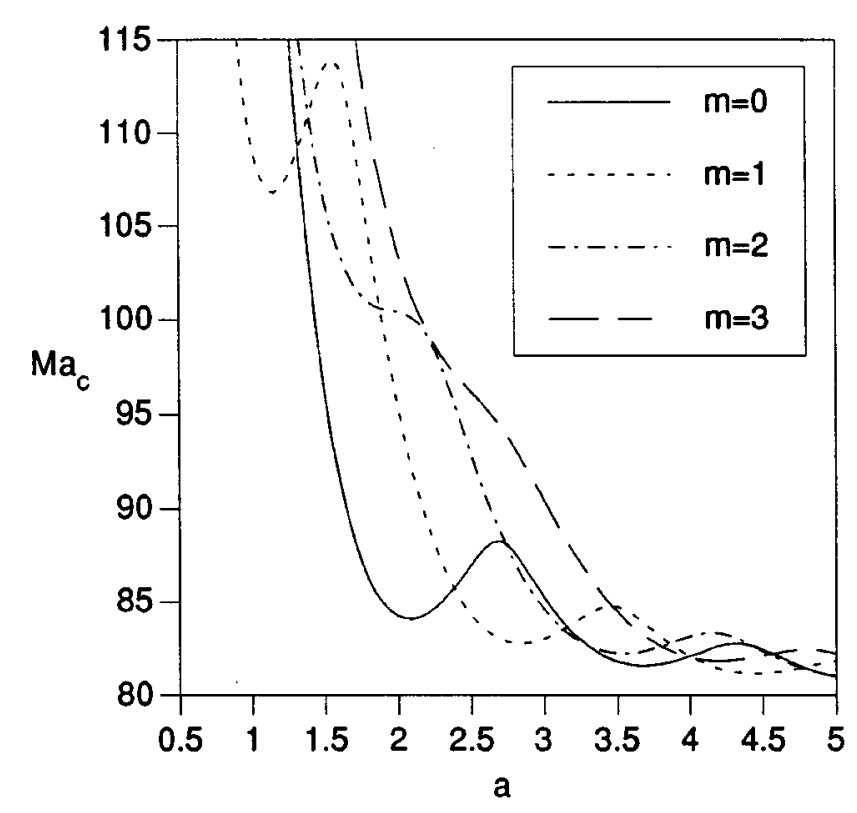

FIG. 1. Critical Marangoni number $\mathrm{Ma}_{c}$ as a function of the aspect ratio $a$ for insulating side walls. Curves corresponding to different azimuthal wave numbers $m$ are represented. The Rayleigh and Biot numbers are zero. 

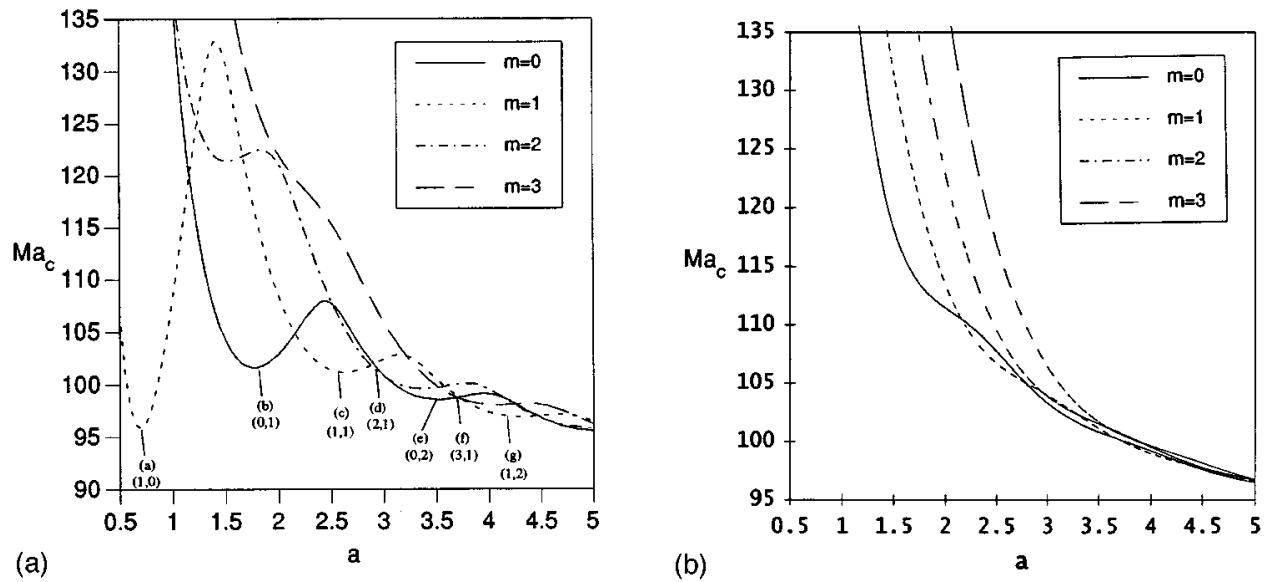

FIG. 2. Critical Marangoni number $\mathrm{Ma}_{c}$ as a function of the aspect ratio $a$ in a container with radius $5.6 \mathrm{~mm}$. A 0.5 -mm gas layer lies above the fluid and the lateral walls are insulating (a) or conducting (b). In (a), small letters indicate aspect ratios for which the convective structure is represented in Fig. 3; the corresponding azimuthal and radial wave numbers $m$ and $i$ are also indicated.

fluid. The wave number $k$ is somewhat undetermined in confined convection, but its importance is minor for small $d_{\text {air }}$ and we will use $k=2$, which is a typical value in natural convection.

Another important point for earth-based experiments is that both the Rayleigh and Marangoni numbers are proportional to the temperature difference so that both of these parameters increase simultaneously in a progressive heating experiment. For this reason, we introduce two dimensionless parameters $\lambda$ and $\alpha$ by the following relations [31]:

$$
(1-\alpha) \frac{\mathrm{Ra}}{\mathrm{Ra}_{0}}=\alpha \frac{\mathrm{Ma}}{\mathrm{Ma}_{0}} \text { and } \lambda=\frac{\mathrm{Ra}}{\mathrm{Ra}_{0}}+\frac{\mathrm{Ma}}{\mathrm{Ma}_{0}},
$$

where $\mathrm{Ra}_{0}$ and $\mathrm{Ma}_{0}$ are two arbitrary constants. Here we fix $\mathrm{Ra}_{0}=669$ and $\mathrm{Ma}_{0}=79.6$. These two values correspond respectively to the critical values of pure gravity-driven and pure capillary convection in a horizontally infinite fluid layer with $\mathrm{Bi}=0$. The parameter $\lambda$ is proportional to the temperature difference and will be considered as the eigenvalue parameter while $\alpha$, which is independent of $\Delta T$, can be interpreted as the percentage of the buoyancy effect with respect to the capillary effect. This parameter can be expressed as $\alpha=\left[1+\mathrm{Ra}_{0} \gamma\left(\mathrm{Ma}_{0} \alpha_{T} \rho g d^{2}\right)^{-1}\right]^{-1}$ and depends on the fluid material properties and on the thickness of the liquid layer. Thick fluid layers correspond to large $\alpha$ and to mainly buoyancy-driven convection while thin films give rise to small $\alpha$ and to capillary instability.

In the experiments of Koschmieder and Prahl, the aspect ratio of the container is varied by modifying the thickness of the fluid layer. It is then clear that $\alpha$ is different in all experiments. Similarly, the Biot number defined in Eq. (4.1) also varies with $d$. In view of these observations we have computed the critical value of $\lambda$ for aspect ratios between 0.5 and 5 by considering the corresponding variations of $\mathrm{Bi}$ and $\alpha$. These parameters are determined by using the material coefficients given in $[6,28]$. The case of a circular cylinder with radius $5.6 \mathrm{~mm}$ is considered in the following. Some typical values of the Biot number for this container are 1.84, 0.66 , and 0.46 for aspect ratios equal to 1,3 , and 5 , respectively.

In Fig. 2, we plot the critical Marangoni number as a function of the aspect ratio for this container and for both insulating [Fig. 2(a)] and conducting side walls [Fig. 2(b)]. For insulating side walls, it is interesting to notice that $\mathrm{Ma}_{c}$ is not globally decreasing since the first portion of the curve lies below the next ones. This is due to the fact that, in small aspect ratio boxes, the instability is mainly gravity driven. In larger boxes, the Marangoni effect is dominant and the critical Rayleigh number tends to zero.

The convective patterns appearing at threshold depend on the aspect ratio, and, in particular, depend on the azimuthal wave number $m$ giving rise to the absolute minimum of the different curves in Fig. 2. The classification of the possible patterns is also made easier by defining a radial wave number $i$. To define this number, we note first that each curve corresponding to a fixed $m$ in Fig. 2 is made up of different regions in which the concavity is successively upwards and downwards. This is most easily seen in Fig. 2(a) where local minima and maxima appear. Then, every region with upwards concavity can be associated with a radial wave number $i$, which is obtained by counting the successive upwards concavity regions from the left of the picture: on a curve with fixed $m$, the first upwards concavity region has a radial wave number $i=0$, the next corresponds to $i=1$, etc. Note, however, that, for $m=0$ the $i=0$ region does not exist for a reason that is explained below.

Typical convective patterns corresponding to insulating side walls are shown in Fig. 3 for different aspect ratios. The vertical velocity $w$ at mid-depth of the container is represented. On each picture, the corresponding radial and azimuthal wave numbers are given. The azimuthal wave number $m$ is the number of times $w$ vanishes for any $r$ within the $\theta$ interval $] 0,2 \pi[$. The radial wave number $i$ can also be seen as the number of times $w$ is equal to 0 within the $r$ interval ] $0,1[$, that is, the number of "radial rolls" that make up the structure. We can now better understand why the mode $(m, i)=(0,0)$ is not present in Fig. 2. This mode would correspond to a pure thermal mode, with zero velocity and uniform horizontal temperature. This mode is the well-known " 0 mode" [31] and is never marginally stable since its growth rate is always negative (when $\mathrm{Ra}=\mathrm{Bi}=0$, for instance, this growth rate is equal to $-\pi^{2} / 4$ ). 

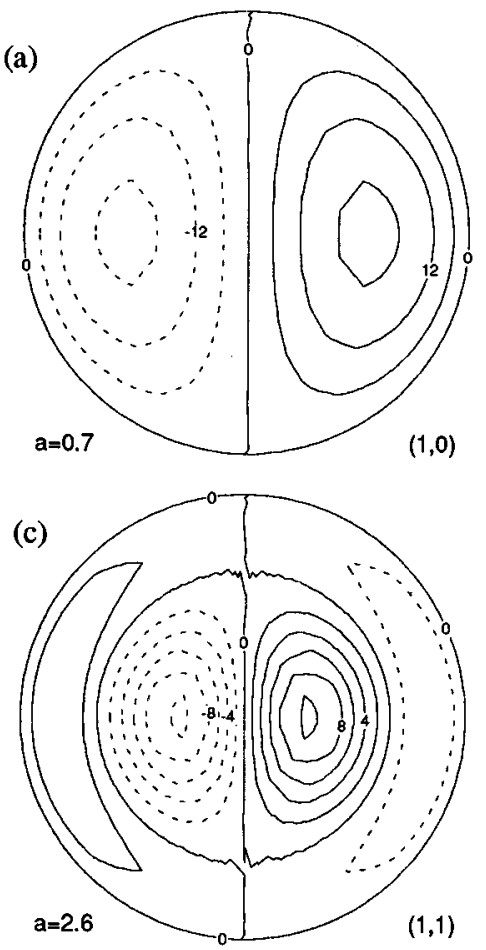

$(1,1)$
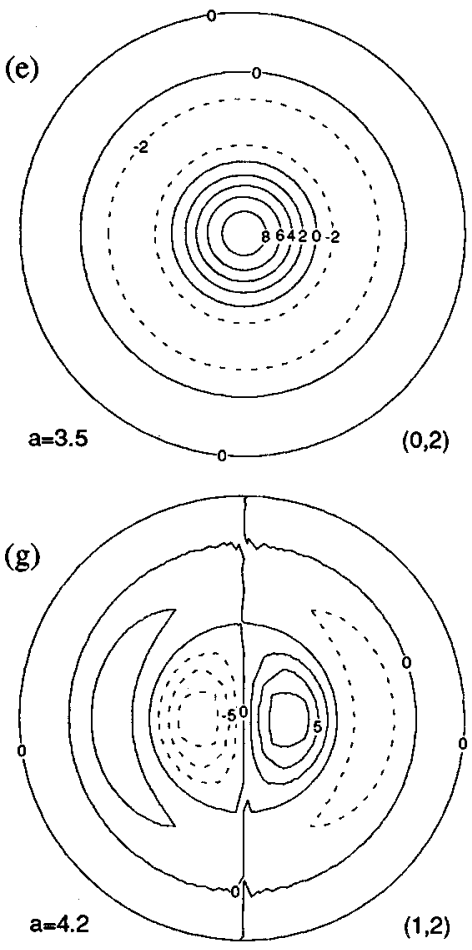

$(1,2)$
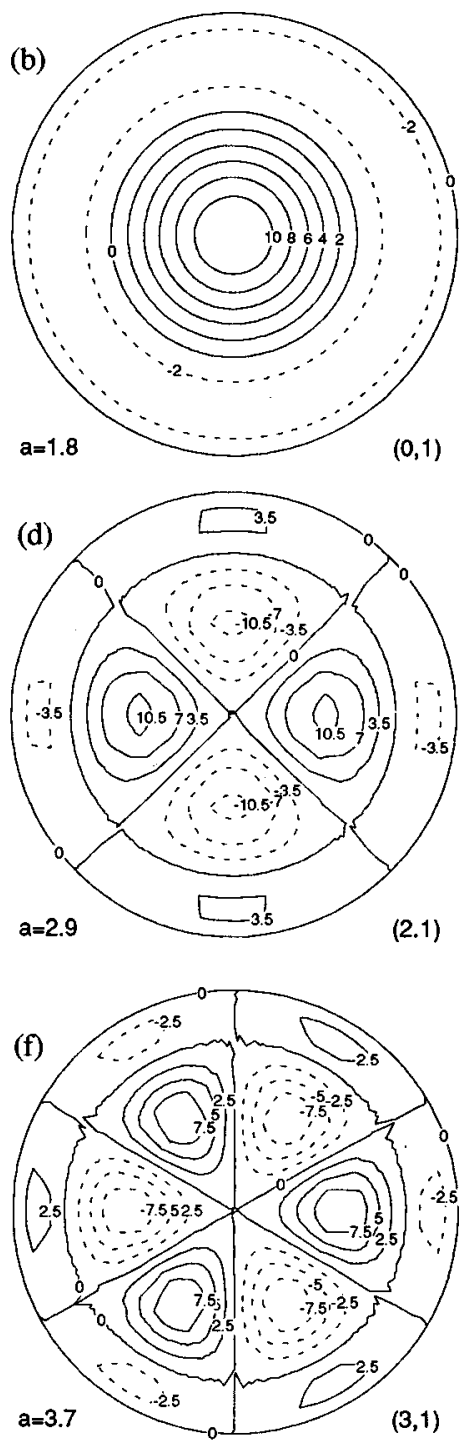

FIG. 3. Convective patterns at the threshold for different aspect ratios. The vertical velocity $w$ at mid-depth of the container is represented. The aspect ratios and the critical azimuthal and radial wave numbers are indicated. The letters (a)-(f) correspond to the labels in Fig. 2(a).
The main results about the convective patterns at the threshold are the following. For very small aspect ratios, the critical mode is the mode $m=1, i=0$. For simplicity, this mode is denoted $(1,0)$. A picture of the convective structure is given in Fig. 3(a) for $a=0.7$. We observe an upflow in the right-hand half of the container and a symmetrical downflow in the left-hand part. For aspect ratios around 1.8, the pattern is axisymmetric and consists of a circular roll centered in the middle of the box [Fig. 3(b)]. For $a$ close to 2.5, the critical mode is the mode $(1,1)$, which is represented in Fig. 3(c). The structure may be seen as made up by three transverse and somewhat deformed rolls. In a very small area around $a=2.9$, the critical pattern has the form given in Fig. 3(d); the marginally stable mode is the mode $(2,1)$. Near $a=3.4$, the critical mode is the mode $(0,2)$, which is also axisymmetric [Fig. 3(e)] and consists of two concentric rolls. When the aspect ratio is very close to 3.7 , the mode $(3,1)$, drawn in Fig. 3(f), is linearly unstable. The last mode represented in Fig. $3(\mathrm{~g})$ corresponds to $a=4.15$; the wave numbers are in this case $(m, i)=(1,2)$ and 5 deformed transverse rolls are displayed. The critical modes for larger values of the aspect ratios are not given because they are easily derived when the wave numbers are known. Moreover, for large aspect ratios, many modes are nearly critical at threshold and it is not 
always easy to determine which one is unstable first.

Let us mention that the succession of the different critical modes when $a$ is increased is similar in Fig. 1 and in Fig. 2(a) even if the aspect ratios for which the switchings occur are somewhat different. Similarly, we observed the same succession when $\mathrm{Ma}_{c}$ is calculated for $\mathrm{Ra}=0, \mathrm{Bi}=2$, for $\mathrm{Ra}=200, \mathrm{Bi}=0$ and also when we calculated $\lambda_{c}$ for the experiments of Koschmieder and Prahl in containers with radii 8.75 and $11.9 \mathrm{~mm}$. In summary, we can say that an increase of $\mathrm{Ra}$ gives rise to a decrease of $\mathrm{Ma}_{c}$ but no significant variation of the pattern is observed. An increase of the Biot number makes the critical Marangoni number larger since perturbations may be dissipated in the upper gas layer. In this case, the cells become smaller and smaller as $\mathrm{Bi}$ increases but the succession of the patterns for increasing aspect ratios remains the same. So, it may be stated that gravity and the Biot number do not seem very important for the succession of the eigenmodes.

The patterns for conducting side walls will not be studied in detail since, for any value of the azimuthal wave number $m$, they are quite similar to those given in Fig. 3. Note, however, that the succession of the critical modes is different since in the conducting case, modes $m=2$ [Fig. 3(d)] and $m=3$ [Fig. 3(f)] are never observed at the threshold.

The succession of patterns when $a$ becomes larger and larger can be summarized in the following way. Except for the structures described by Fig. 3(d) and Fig. 3(f), which appear only for insulating side walls and for a very small range of the aspect ratio, the patterns observed at the threshold are made up by concentric rolls or by some kind of deformed transverse rolls. In very small boxes, a unique transverse roll is observed [Fig. 3(a)]. Then, when $a$ is increased, a unique roll with its center in the middle of the vessel appears [Fig. 3(b)]. Figure 3(c) exhibits 3 transverse rolls while 2 concentric rolls are displayed in Fig. 3(e). Finally, 5 transverse rolls are present in Fig. 3(g). So, the number of rolls is progressively increased. The number of transverse rolls is always odd while the number of concentric rolls may take any value.

A last comment on the radial wave number $i$ is in order. It is important to notice that along a curve with fixed azimuthal wave number $m$ in Fig. 2, the variations of the convective structure are continuous. So a radial wave number is not easy to define everywhere along these curves. For instance, the $m=0$ convective pattern in the neighborhood of $a=2.5$ in Fig. 2(a) shows a continuous transition from $i=1$ to $i=2$. For aspect ratios just on the right of the minimum at $a$ $=1.7$ for the $m=0$ curve in Fig. 2(a), two small and very weak axisymmetric rolls appear along the side walls, one at the top surface and the other one at the bottom of the container. When the aspect ratio still increases, these two rolls grow and eventually merge to give birth to the $i=2$ mode (see also [25]).

In view of a future nonlinear approach of the problem, it is worth making some comments on the symmetry of the different patterns [32-35]. First we can note that all curves in Figs. 1 and 2 are crossing each other, which is made possible because the eigenmodes corresponding to these different curves have different azimuthal wave numbers and thus different symmetries. On the other hand, if the successive increasing eigenvalues for each $m$ had been presented, the different curves corresponding to a fixed azimuthal wave number could not cross because the eigenmodes have the same $\theta$ symmetry. Some further remarks may be added concerning the bifurcation of the different eigenmodes. First, the $m=0$ eigenmodes are axisymmetric and are also invariant for a reflection about any diameter of the box. The bifurcation of such modes is nondegenerate and transcritical for the Bénard-Marangoni problem. On the other hand, the bifurcation of eigenmodes with nonvanishing azimuthal wave numbers is degenerate and a complete family of solutions emerges symmetrically at the bifurcation point, each member being characterized by its azimuthal phase. The transcritical or symmetric character of the bifurcation for axisymmetric or nonaxisymmetric modes, respectively, was also noticed in the numerical study of Wagner et al. [27].

To finish this section, we discuss briefly the principle of exchange of stability. Our code has the possibility to consider the growth rate of the perturbations as the eigenvalue parameter when $\mathrm{Ra}$ and $\mathrm{Ma}$ are fixed. The validity of the principle of exchange of stability has then been checked in the following way. First, a zero growth rate is fixed and the critical $\mathrm{Ra}_{c}$ and $\mathrm{Ma}_{c}$ are determined. Then the growth rate is recalculated with fixed $\mathrm{Ra}=\mathrm{Ra}_{c}$ and $\mathrm{Ma}=\mathrm{Ma}_{c}$. The result is then that, in all cases, the growth rate with the largest real part is actually 0 . This analysis provides thus a numerical proof of the validity of the principle of exchange of stability in the circular Bénard-Marangoni problem for both insulating and conducting side walls.

\section{COMPARISON WITH OTHER THEORETICAL WORKS}

As already mentioned in the Introduction, several papers on the same problem exist, whose results are now compared with ours.

A linear study of thermoconvective instabilities in circular bounded domains was first presented by Vrentas, Narayanan, and Agrawal in 1981 for axisymmetric perturbations [25]. It is easy to compare our $m=0$ results with those of these authors. Some typical situations are analyzed in Table III, which shows excellent agreement between both approaches (difference nearly always less $0.1 \%$ ), except for the critical Rayleigh numbers when the aspect ratio is equal to 1 . This disagreement for the Rayleigh problem with small $a$ is somewhat surprising and we have rechecked the convergence of our results in this case (for $\mathrm{Bi}=1$, we got $\mathrm{Ra}_{c}=1483.11$ with $N_{r} \times N_{z}=4 \times 4$ and $\mathrm{Ra}_{c}=1481.84$ with $\left.N_{r} \times N_{z}=20 \times 20\right)$. Actually, we have also checked the other results given in Vrentas et al. Except for the Rayleigh problem with aspect ratios lower than or equal to 1 , the agreement was excellent.

The comparison of the critical numbers with the work by Chen et al. [26] is not easy because their results are presented only graphically. Moreover, we have already mentioned that their axisymmetric results are probably incorrect. However, except for the $m=0$ mode, it is interesting to note that the succession of the modes in their Fig. 2 is the same as in our Fig. 1, with codimension-2 points appearing for values of the aspect ratios close to ours.

The numerical results given in Table I of Wagner et al. have also been compared to our calculations. The deviations between the 4 numbers they give and ours are, respectively, $0.3,3,2$, and $6 \%$; the agreement can thus be considered as 
TABLE III. Comparison between our results (D. et al.) and those of Vrentas et al. (V. et al.) [25]. The first lines give the critical Marangoni number when $\mathrm{Ra}=0$ and for different Biot numbers; the bottom of the table presents the critical Rayleigh number for $\mathrm{Ma}=0$. Note that the values of Vrentas et al. have been multiplied by $\mathrm{Bi} /(\mathrm{Bi}+1)$ in order to correspond to our notation.

\begin{tabular}{|c|c|c|c|c|c|c|}
\hline \multirow[b]{2}{*}{$\mathrm{Bi}(\mathrm{Ra}=0)$} & \multicolumn{2}{|c|}{$a=1$} & \multicolumn{2}{|c|}{$a=2$} & \multicolumn{2}{|c|}{$a=4$} \\
\hline & $\begin{array}{c}\mathrm{Ma}_{c} \\
\text { (D. et al.) }\end{array}$ & $\begin{array}{c}\mathrm{Ma}_{c} \\
\text { (V. et al.) }\end{array}$ & $\begin{array}{c}\mathrm{Ma}_{c} \\
\text { (D. et al.) }\end{array}$ & $\begin{array}{c}\mathrm{Ma}_{c} \\
\text { (V. et al.) }\end{array}$ & $\begin{array}{c}\mathrm{Ma}_{c} \\
\text { (D. et al.) }\end{array}$ & $\begin{array}{c}\mathrm{Ma}_{c} \\
\text { (V. et al.) }\end{array}$ \\
\hline 0.01 & 164.55 & 164.40 & 84.638 & 84.635 & 82.486 & 82.483 \\
\hline 0.1 & 168.34 & 168.35 & 88.344 & 88.264 & 86.263 & 86.582 \\
\hline 1 & 206.16 & 206.00 & 125.01 & 125.0 & 120.63 & 120.65 \\
\hline $\mathrm{Bi}(\mathrm{Ma}=0)$ & $\begin{array}{c}\mathrm{Ra}_{c} \\
\text { (D. et al.) }\end{array}$ & $\begin{array}{c}\mathrm{Ra}_{c} \\
\text { (V.et al.) }\end{array}$ & $\begin{array}{c}\mathrm{Ra}_{c} \\
\text { (D. et al.) }\end{array}$ & $\begin{array}{c}\mathrm{Ra}_{c} \\
\text { (V. et al.) }\end{array}$ & $\begin{array}{c}\mathrm{Ra}_{c} \\
\text { (D. et } a l .)\end{array}$ & $\begin{array}{c}\mathrm{Ra}_{c} \\
(\mathrm{~V} . \text { et } a l .)\end{array}$ \\
\hline 0.01 & 1419.47 & 1558.3 & 712.667 & 712.466 & 695.668 & 695.548 \\
\hline 0.1 & 1426.24 & 1565.9 & 726.704 & 726.482 & 709.457 & 709.336 \\
\hline 1 & 1482.12 & 1628.2 & 836.072 & 835.85 & 799.735 & 799.5 \\
\hline
\end{tabular}

satisfactory since the numbers given by Wagner et al. are obtained by interpolating the bifurcation points from the nonlinear regime.

The agreement between our work and that of Zaman and Narayanan can be tested by comparing our Table II (for insulating side walls) with their Table II. For aspect ratios equal to 1 or 2 , the difference between both approaches may reach $13 \%$ or $12 \%$ but it is important to stress that the azimuthal wave number of the most unstable mode is the same in both papers $(m=1$ for $a=1$ and $m=0$ for $a=2)$. For $a$ $=4$ or $a=8$, the deviations are far less (less than $2 \%$ ) but the critical $m$ 's are different in both approaches. More generally, we have checked that the succession of the eigenmodes when $a$ is increased is not the same in both approaches. In particular, the critical $m=2$ zone in the neighborhood of $a=1.2$ in Fig. 3 of Zaman and Narayanan's paper does not appear in our Fig. 2.

It is not easy to guess the reason for this disagreement. In their paper, Zaman and Narayanan do not discuss the regularity conditions at $r=0$, but these seem to be satisfied by their trial functions, at least for $m \neq 1$. Probably, the difference between our work and those of Vrentas et al. and of Zaman and Narayanan originate in the numerical methods, which are not exactly similar and which could be more or less efficient, depending on the aspect ratio or on the type of the problem under consideration (Marangoni, Rayleigh, or coupled problem) [28,37].

Comparison with the work of Rosenblat et al. [13] is not easy since the lateral vorticity-free wall assumption used by these authors is quite different from our more realistic model of no-slip boundaries. It is, however, worth noting that the artifact that some finite containers have the same critical value for the control parameter as infinite layers disappears with rigid side walls. Moreover, the succession of the critical modes when the aspect ratio is increased in [13] is also quite different and many critical modes predicted in slippery containers are never observed at the threshold with a no-slip condition. This reduction of the possible patterns observed at criticality is due to the continuous variation of the structure along a curve with a fixed azimuthal wave number $m$. In the case of rectangular boxes, a similar behavior was also observed in [24].

\section{COMPARISON WITH EXPERIMENTS}

Zaman and Narayanan [25] have already considered in detail the comparison between experiments and a linear approach to the stability problem with conducting side walls. In particular, they propose several arguments to explain why the calculated critical values of the control parameters are usually $20 \%$ higher than the experimental measurements of Koschmieder and Prahl [6]. Let us add that this difference can be slightly decreased by considering insulating side walls (Koschmieder and Prahl used bakelite for the side walls), which make the system more unstable but the improvement is minor. Note also that the assumption of a passive gas above the fluid may have some influence on the discrepancy between theoretical predictions and experimental measurements of the thresholds [38]. This point will not be discussed in more detail here but we would like to add some comments on the convective patterns. The main point we want to stress now is that, in most cases, the linear study of the pattern does not provide a good description of what is experimentally observed, whatever the insulating or conducting character of the side walls. Actually, the comparison of Fig. 1 in [6] with our Fig. 3 shows that the observed patterns are different from the structures we deduced from our analysis, except perhaps the axisymmetric pattern of Fig. 1(a) in [6], which is the one-circular roll solution depicted in our Fig. 2(b). In fact, we already stressed this disagreement between linear theory and experiments in a previous work on confined thermoconvection in rectangular vessels [24]. It was shown in this work that the convective pattern in the nonlinear regime may be quite different from the pattern predicted at the threshold, especially for large containers. The reason is twofold. First, the modes that are generated by the self-interactions of the critical mode in the nonlinear regime do not always have a small amplitude so that their presence may deeply influence the final pattern, which is actually the superposition of the linearly unstable structure and these nonlinearly generated modes. Second, and this was also 
mentioned by Zaman and Narayanan, some stable, and thus observable, solutions in the nonlinear regime correspond to branches that do not emanate from the lowest bifurcation point on the Ma axis. This is particularly true in large boxes in which the successive eigenmodes bifurcate quite near the threshold: Fig. 2 shows indeed that the curves are quite near each other on the right side of the picture. In small boxes, the curves for the successive eigenmodes are well separated and the secondary modes should not be observed in the neighborhood of the threshold.

\section{SUMMARY}

By using a spectral tau method, we have analyzed the linear Bénard-Marangoni problem in circular rigid containers. We have shown that the principle of exchange of stability is valid so that the linear instability is stationary. The numerical convergence of our method has been analyzed. In particular, we have checked that the critical parameters for confined geometries remain always larger than in infinite domains and tend to Nield's values for large vessels. We have also verified that the system is more stable with conducting lateral boundaries than with insulating side walls.

The main results are summarized in Figs 2 and 3. Figure 2 describes the succession of the possible patterns when the aspect ratio is increased. This succession differs slightly for insulating and conducting side walls but is independent of the Biot number and of the ratio of the Rayleigh and Marangoni numbers. Figure 3 gives pictures of the convective motions, which generally take the form of either concentric or "transverse" cells. Eventually, comparisons with previous analyses and with experiments are also discussed.

\section{ACKNOWLEDGMENTS}

This text presents research results of the Belgian Interuniversity Poles of Attraction (PAI No. 4/06) initiated by the Belgian State, Prime Minister's Office, Science Policy Programming. The scientific responsibility is assumed by its authors. Partial support from the European Community under Contract No. ERB-CHRX-CT94-0481 is also acknowledged. It is a pleasure to thank our colleagues P. Parmentier and V. Regnier and Professor P. Cerisier from the University of Marseille for stimulating discussions. We are also grateful to Professor J.-C. Legros and his group (Brussels University) for interesting comments. Part of this work was completed during a stay of one of us (P.C.D.) in the group of Professor R. Narayanan at the University of Florida at Gainesville. It is a pleasure to thank him and D. Johnson for their kind hospitality and their very interesting suggestions, which really have contributed to the improvement of this work. The FNRS ("Fonds National de la Recherche Scientifique," Belgium) and the "Patrimoine de l'Université de Liège" are also acknowledged for the financial support that allowed this stay.

\section{APPENDIX}

In this Appendix, we present some details on the numerical method used to solve the linear eigenvalue problem.

First we present the trial functions to be used in the spectral method. The undefined symbols in Eq. (3.1) are given by

$$
\begin{gathered}
u_{i k}^{r}=f_{i}(r) \partial_{z} g_{k}(z), \quad w_{i k}^{r}=a^{-1} u_{i}(r) g_{k}(z), \\
v_{i k}^{\theta}=m f_{i}(r) \partial_{z} g_{k}(z), \quad w_{i k}^{\theta}=m^{2} a^{-1} v_{i}(r) g_{k}(z), \\
u_{k}^{0}=F(r) \partial_{z} g_{k}(z), \quad v_{k}^{0}=-F(r) \partial_{z} g_{k}(z), \\
w_{k}^{0}=a^{-1} U(r) g_{k}(z) \\
\theta_{i k}=m_{i}(r) n_{k}(z)
\end{gathered}
$$

where $\partial_{z}$ denotes derivatives with respect to $z$. The functions $f_{i}, u_{i}, v_{i}, F, U, m_{i}, g_{k}$, and $n_{k}$ are selected in order that (A1)-(A4) form a complete set but also in such a way that most of the boundary and regularity conditions are satisfied $a$ priori. Here, we consider products of shifted Chebyshev polynomials (defined on $[0,1]$ ) and other polynomials that allow one to take the boundary and regularity conditions into account. The trial functions $g_{k}$ and $n_{k}$, which depend on the vertical coordinate $z$, are independent of the value $m$ of the azimuthal wave number. These $z$ functions are written for $k=1, \ldots, N_{z}$ as

$$
g_{k}(z)=z^{2}(z-1) T_{k-1}(z), \quad n_{k}(z)=z T_{k-1}(z)
$$

where $T_{k}$ 's are the shifted Chebyshev polynomials defined on $[0,1]$.

With this choice for the $z$ functions, it is easy to check that the zero velocity and the fixed temperature conditions on the rigid bottom wall are identically satisfied. The nondeformability condition at the top surface is also ensured.

The functions $u_{i}, v_{i}$, and $U$ are given by

$$
\begin{gathered}
u_{i}=-r^{-1} \partial / \partial r\left(r f_{i}\right), \quad v_{i}=-r^{-1} f_{i}, \\
U=-\partial F / \partial r .
\end{gathered}
$$

Because of these relations, it is easily checked that the incompressibility condition (2.2) is automatically fulfilled.

The horizontal trial functions $f_{i}, F$, and $m_{i}$ depend on the azimuthal wave number $m$. For $m=1$, one has

$$
\begin{gathered}
f_{i}(r)=r^{2}(r-1)^{2} T_{i-1}(r), \quad F(r)=\left(r^{2}-1\right)^{2}, \\
m_{i}(r)=\int_{0}^{r}(\xi-1) T_{i-1}(\xi) d \xi(\mathrm{ISW}), \\
m_{i}(r)=r(r-1) T_{i-1}(r) \quad(\mathrm{CSW}) .
\end{gathered}
$$

With the above choice for the trial functions, it is easy to verify that the zero velocity condition is automatically ensured as well as the regularity conditions. Indeed, the temperature perturbations actually vanish at $r=0$; the terms proportional to $A_{i k}$ and $B_{i k}$ vanish for the three components of 
the velocity while the terms proportional to $X_{k}$ allow one to satisfy $u=-v ; w=0$ at $r=0$. So, all the boundary and regularity conditions are a priori satisfied, except for the Marangoni and Biot conditions.

The horizontal trial functions $f_{i}$ and $m_{i}$ for $m>1$ take the same from (A7), but the terms in $X_{k}$ are canceled in Eqs. (A1)-(A3) (the functions $F$ and $U$ are no longer necessary).
Except for the Marangoni and Biot relations, the boundary conditions are directly satisfied as well as the regularity conditions.

The particular case $m=0$ is obtained from Eqs. (A1)(A4) by canceling terms proportional to $X_{k}$ and to $B_{i k}$. In order to fulfill the natural boundary conditions and the regularity conditions, the functions $f_{i}$ and $m_{i}$ are selected as

$$
f_{i}(r)=r(r-1)^{2} T_{i-1}(r),\left\{\begin{array}{l}
m_{1}(r)=1 \\
m_{i>1}(r)=\int_{0}^{r}(\xi-1) T_{i-2}(\xi) d \xi
\end{array}(\text { ISW }), m_{i}(r)=(r-1) T_{i-1}(r) \quad(\mathrm{CSW}) .\right.
$$

The spectral tau method consists in introducing the general expressions (A1)-(A4) with Eqs. (A5)-(A8) in Eqs. (2.3)-(6) and the boundary conditions (2.9)-(11). The algebraic equations are obtained by projection on the trial functions, that is, by multiplying the equations by the trial functions and integrating the products over the fluid volume. The method is similar to that used in [24] and will not be commented upon in detail. The whole set of equations forms an algebraic eigenvalue problem for $\mathrm{Ma}$ and/or $\mathrm{Ra}$. The dimension of the eigenvalue problem is $3 \times N_{r} \times N_{z}$ for an azimuthal wave number $m$ larger than 1 . For $m=1$ and $m=0$, the dimensions are respectively $\left(3 \times N_{r}+1\right) \times N_{z}$ and 2 $\times N_{r} \times N_{z}$. The calculations have been carried out using the
ESSL routine DGEGV. Note eventually that all singularities at $r=0$ due to factors $r^{-1}$ or $r^{-2}$ in (2.2)-(2.6), (2.10), (2.11), or (A6) disappear from the final projected equations as a result of the regularity conditions, which are a priori satisfied by our trial functions.

Note also that the normalization condition used to determine the algebraic eigenvectors is the following. The temperature perturbation is calculated at mid-depth of the layer for $r$ equal to $0.25,0.5,0.75$, and 1 . The point for which $|\theta|$ is maximum is then chose to fix $\theta=1$. This normalization condition is of importance for the nonlinear analysis to be developed in a future work but is of no concern in a linearized approach.
[1] E. L. Koschmieder, Bénard Cells and Taylor Vortices (Cambridge University Press, Cambridge, 1993).

[2] H. Bénard, Rev. Gen. Sci. Pure Appl. 11, 1261 (1900).

[3] Lord Rayleigh, Philos. Mag. 32, 529 (1916).

[4] J. R. A. Pearson, J. Fluid Mech. 4, 489 (1958).

[5] D. A. Nield, J. Fluid Mech. 19, 341 (1964).

[6] E. L. Koschmieder and S. A. Prahl, J. Fluid Mech. 215, 571 (1990).

[7] A. B. Ezersky, A. D. Preobrazhensky, and M. I. Rabinovich, Eur. J. Mech. B, Fluids, 10, 205 (1991).

[8] T. Ondarçuhu, J. Millán-Rodríguez, H. L. Mancini, A. Garcimartín, and C. Pérez-García, Phys. Rev. E 48, 1051 (1993).

[9] T. Ondarçuhu, G. B. Mindlin, H. L. Mancini, and C. PérezGarcía, Phys. Rev. Lett. 70, 3892 (1993).

[10] D. Johnson and R. Narayanan, Phys. Rev. E 54, R3102 (1996).

[11] D. Schwabe, O. Dupont, P. Queeckers, and J. C. Legros (unpublished).

[12] S. H. Davis, J. Fluid Mech. 30, 465 (1967).

[13] S. Rosenblat, S. H. Davis, and G. M. Homsy, J. Fluid Mech. 120, 91 (1982).

[14] S. Rosenblat, G. M. Homsy, and S. H. Davis, J. Fluid Mech. 120, 123 (1982).

[15] P. C. Dauby, G. Lebon, P. Colinet, and J. C. Legros, Q. J. Mech. Appl. Math. 46, 683 (1993).
[16] B. Echebarría, D. Krmpotic, and C. Pérez-García, Physica D 99, 487 (1997).

[17] D. Johnson and R. Narayanan (unpublished).

[18] K. H. Winters and Th. Plesser, Physica D 29, 387 (1988).

[19] A. I. van de Vooren and H. A. Dijkstra, Comput. Fluids 17, 467 (1989).

[20] H. A. Dijkstra, J. Fluid Mech. 243, 73 (1992).

[21] H. A. Dijkstra, Microgravity Sci. Technol. VII, 307 (1995).

[22] H. A. Dijkstra, Microgravity Sci. Technol. VIII, 70 (1995).

[23] H. A. Dijkstra, Microgravity Sci. Technol. VIII, 155 (1995).

[24] P. C. Dauby and G. Lebon, J. Fluid Mech. 329, 25 (1996).

[25] J. S. Vrentas, R. Narayanan, and S. S. Agrawal, Int. J. Heat Mass Transf. 24, 1513 (1981).

[26] J.-C. Chen, J.-Y. Chen, and Z.-C. Hong (unpublished).

[27] C. Wagner, R. Friedrich, and R. Narayanan, Phys. Fluids 6, 1425 (1994).

[28] A. A. Zaman and R. Narayanan, J. Colloid Interface Sci. 179, 151 (1996).

[29] C. Canuto, M. Y. Hussaini, A. Quarteroni, and T. A. Zang, Spectral Methods in Fluid Dynamics (Springer-Verlag, Berlin, 1988).

[30] C. Normand, Y. Pomeau, and M. Velarde, Rev. Mod. Phys. 49, 581 (1977).

[31] P. Parmentier, V. Regnier, G. Lebon, and J.-C. Legros, Phys. Rev. E 54, 411 (1996). 
[32] M. Golubitsky and D. G. Schaeffer, Singularities and Group in Bifurcation Theory (Springer, New York, 1985), Vol. 1.

[33] M. Golubitsky, I. Steward, and D. G. Schaeffer, Singularities and Group in Bifurcation Theory (Springer, New York, 1988), Vol. 2.

[34] J. D. Crawford and E. Knobloch, Annu. Rev. Fluid Mech. 23, 341 (1991).
[35] K. A. Cliffe and K. H. Winters, J. Comput. Phys. 67, 310 (1986).

[36] A. X. Zhao, F. C. Moates, and R. Narayanan, Phys. Fluids 7, 1576 (1995).

[37] R. Narayanan (private communication).

[38] D. Johnson, R. Narayanan, and P. C. Dauby (unpublished). 